\title{
Potential Molecular Mechanisms for Improved Prognosis and Outcome with Neoadjuvant Chemotherapy Prior to Laparoscopical Radical Hysterectomy for Patients with Cervical Cancer
}

\author{
Hongzan Sun ${ }^{\mathrm{a}}$ Jun Xin ${ }^{\mathrm{a}}$ Zaiming Lu Ning Wang $^{\mathrm{b}} \quad$ Ning Liu $^{\mathrm{b}} \quad \mathrm{Qiyong}_{\mathrm{Guo}}^{\mathrm{a}}$
}

aDepartment of Radiology, Shengjing Hospital of China Medical University, Shenyang, bepartment of Gynecology and Obstetrics, Shengjing Hospital of China Medical University, Shenyang, Liaoning, China

\section{Key Words}

Cervical cancer • Neoadjuvant chemotherapy (NAC) • miRNAs • miR-34a • miR-605 • p53 • $\mathrm{E} 2 \mathrm{~F} \cdot \mathrm{Mdm} 2 \cdot$ Cisplatin $\bullet$ Paclitaxel $\bullet$ Carboplatin • ERK1/2 • TAB1

\begin{abstract}
Background: The p53:miR-34a:E2F positive feed-forward loop and the p53:miR-605:Mdm2 positive feed-back loop have been identified to be crucial oncogenesis/tumor suppressorregulating signaling pathways. In this study, we sought to examine the hypothesis that neoadjuvant chemotherapy (NAC) is a better approach with improved prognosis and outcomes after laparoscopical radical hysterectomy $(\mathrm{LRH})$ on patients with cervical cancer and to elucidate the potential roles of the p53:miR-34a:E2F1 and the p53:miR-605:Mdm2 signaling pathways in this therapy. Methods: Twenty-one patients with stage IIB cervical cancer were recruited to this study and they were randomly divided into two groups: $\operatorname{LRH}(n=10)$ and $\mathrm{NAC}+\mathrm{LRH}(\mathrm{n}=11)$ groups. The NAC+LRH group consisted of 4 cycles of cisplatin, paclitaxel and carboplatin. Complication rates and NAC outcomes (tumor size changes, 2-year diseasefree survival rate, and 2-year overall survival rate) were compared between the two groups. Expression of p53, Mdm2, E2F1, miR-34a, and miR-605 at mRNA and protein levels from the tumor tissues was analyzed. Results: We observed that the diameter of tumors following chemotherapy was substantially smaller in the NAC+LRH patients than in LRH patients. No recurrence or metastasis after surgery was observed in the NAC+LRH patients, whereas 2 out of $10 \mathrm{LRH}$ patients had recurrences and 1 had metastasis. The 2-year disease-free and overall survival rates were apparently higher in the NAC+LRH group than in the LRH group. Furthermore, molecular biology analyses revealed that the protein and mRNA levels of p53 were both markedly increased in patients who received NAC than those who did not, and oppositely, the levels of E2F1 and Mdm2 were significantly lower in the NAC+LRH patients than in the LRH patients. The levels of miR-34a and miR-605 were considerably higher with

Qiyong Guo, MD, PhD

Department of Radiology, Shengjing Hospital of China Medical University Shenyang, Liaoning 110004 (China)

Tel. +86-4-96615-7325, E-Mail guoqy_cmu@sina.com
\end{abstract}


NAC relative to without NAC. Among the three anti-cancer drugs included in NAC, cisplatin was found to be the main component that caused increases in 153 protein levels, miR-34a and miR-605 miRNA levels, and decreases in Mdm2 and E2F1 protein levels. Furthermore, ERK1/2 inhibitor U0126 or TAB1 siRNA mitigated these changes induced by cisplatin. Conclusion: These findings not only indicate NAC as a rational approach for better treatment of cervical cancer with improved therapeutic outcomes, due partly to the ability of cisplatin to promote the p53:miR-34a:E2F1 positive feed-forward loop and the p53:miR-605:Mdm2 positive feedback loop.

Copyright $\odot 2013$ S. Karger AG, Basel

\section{Introduction}

Cervical cancer is one of the most common tumors in women with incidence ranking first in female reproductive tract tumors and mortality rate ranking second in various female malignant tumors [1-3]. On a global scale, approximately 500,000 new cases of cervical cancer are reported every year and about 230,000 of them die of this malignancy annually. Histologically, cervical cancer can be divided into squamous cell carcinomas (SCCs; 80\% of cases) and adenocarcinomas (AdCAs; 5-20\% of cases) [4, 5]. Cervical cancer develops through progression from normal cervical epithelium through squamous intraepithelial lesions (SIL) to invasive cancer, which is caused by a persistent infection by the human papillomavirus (HPV). The HPV E6 oncoprotein binds to the tumor suppressor gene product p53, promoting its degradation to reduce its level and activity [6-10]. Consistent with the E6 function of the high-risk HPV types, the majority of cervical cancer cells have a wild-type p53 gene, but the protein levels are strongly decreased.

In developed countries, the majority of patients present with stage I cervical cancer, whereas in developing countries, $~ 80-90 \%$ of cervical cancer patients are at stage III-IV [11, 12]. Stage IB-IIB cervical cancer appears to have a relatively favorable prognosis; however the rate of treatment failure remains as high as $20 \%$. The survival rate with early stage cervical cancer after radical hysterectomy and pelvic lymphadenectomy depends on the presence or absence of several poor prognostic pathologic risk factors. In order to reduce the risks of recurrence, progression or death, neoadjuvant therapy is recommended for these patients [13-16].

MicroRNA (miRNA) signaling pathways have recently been shown to play a critical role in human cancer genesis and progression. Among many miRNAs, the p53:miR-34a:E2F1 positive feed-forward loop [17-19] and the p53:miR-605:Mdm2 positive feed-back loop [20-22] have been identified to be rather aggressive oncogenic signaling pathways in a number of cancers. miR-34 has recently been identified as a direct transcriptional target of p53 [17-19], and downregulation/inactivation of p53, as in the setting of cervical cancer, downregulates miR-34 that in turn increases expression of oncoprotein E2F1 via derepression so as to promote cancer cell proliferation and enhance resistance to apoptosis. On the other hand, in cancers with wild-type p53 status as in cervical cancer, the function of p53 is also inhibited through direct interaction with $\mathrm{Mdm} 2$ oncoprotein, a negative feedback loop to limit the function of p53. In response to oncogenic stimuli, p53 escapes the $\mathrm{p} 53: \mathrm{Mdm} 2$ negative feedback to accumulate rapidly to induce cell cycle arrest and apoptosis. A recent study revealed that miR-605 acts to interrupt p53:Mdm2 interaction to create a positive feedback loop aiding rapid accumulation of p53 to facilitate its function in response to cellular stress via repressing Mdm2 expression [22]. Clearly, enhancing the p53:miR34a:E2F1 positive feed-forward loop and the p53:miR-605:Mdm2 positive feed-back loop should improve anti-cancer therapy.

In this study, we sought to examine the hypothesis that neoadjuvant chemotherapy (NAC) is a feasible and safe approach with improved prognosis and outcomes after laparoscopical radical hysterectomy (LRH) on patients with high and intermediate risk stage IIB cervical cancer and to elucidate the underlying molecular mechanisms, or more specifically, the 
potential roles of the p53:miR-34a:E2F1 positive feed-forward and the p53:miR-605:Mdm2 positive feed-back signaling pathways.

\section{Materials and Methods}

\section{Ethics Statement}

All patients agreed to participate in the study and gave written informed consent. This study was approved by the medical ethics committee of Shengjing Hospital of China Medical University and complied with the Declaration of Helsinki.

\section{Case selection}

A total of 21 patients (average age 45.4, range 33-64) were enrolled between January 2009 and June 2010 in Shengjing Hospital of China Medical University for the current study. All patients underwent physical and gynecologic examination, abdominal and pelvic computed tomography (CT) and/or magnetic resonance imaging (MRI) before NAC. The patients were diagnosed with IIB cervical cancer, according to the 2000 FIGO criteria (International Federation of Gynecology and Obstetrics) [18], and cervical local lesion size was measured by magnetic resonance imaging (MRI). These patients had bulky cervical cancer and were treated with NAC (paclitaxel combined with carboplatin systemic chemotherapy and cervical local injection of cisplatin). Initial treatment and diagnoses of the patients were confirmed by cervical biopsy prior to surgery.

\section{$N A C \& L R H$}

The patients were randomly divided into two groups: LRH $(n=10)$ group and NAC+LRH $(n=11)$ group. The NAC+LRH group was treated with NAC drugs as one treatment course: cisplatin (20 mg, cervical local injection) on day 1 and day 4, paclitaxel (Nanjing Sike Pharmaceutical Co., Ltd.; Nanjing, China) (150 mg/ $\mathrm{m}^{2}$ intravenous infusion) on day 2 , and carboplatin (i.v., $\mathrm{AUC}=5$ ) on day 3 . The treatment course was repeated every 4 weeks. Following NAC, laparoscopic radical hysterectomy (LRH) with pelvic lymphadenectomy was performed. Adjuvant irradiation was given to patients with negative lymph nodes and one or more surgical pathological risk factors (deep stromal invasion, lymph-vascular space involvement, grade 3 differentiation, maximum tumor diameter $>4 \mathrm{~cm}$ and adenocarcinoma histotype).

\section{Evaluation of NAC}

Gynecological examination was carried out prior to each chemotherapy course to assess the lesion development and stage progression. Re-examination of MRI was performed prior to therapy in order to evaluate the efficacy of chemotherapy. The efficacy of NAC was evaluated according to the WHO evaluation criteria: (1) complete remission (CR): tumor disappeared completely with no lymph node metastasis; (2) partial remission (PR): at least 50\% tumor shrinkage; (3) stable disease (SD): tumors shrank by $<50 \%$ or enlarged by $<25 \%$; (4) progression disease (PD): tumors enlarged by $>25 \%$ or there was a new lesion. Possible side-effects were continuously monitored during chemotherapy and were evaluated according to the standard of chemotherapy toxic response issued by the World Health Organization (WHO).

\section{Follow-up}

Out-patient follow-up was carried out in all 21 patients, once every three months after the first year of therapy. Follow-up consisted of gynecological examination, vaginal stump brushing cytology examination, pelvic B-us, B-us examination of kidneys, ureters and bladder, and chest examination.

\section{Surgical specimens}

Cervical tumor tissues were intra-operatively obtained from 10 patients with LRH only and 11 patients with NAC+LRH enrolled into this study. The specimens were immediately frozen in liquid nitrogen for measuring mRNA and protein levels of selected genes, except for a portion which was used for hematoxylin and eosin (H\&E) staining and pathological examination to confirm the diagnosis. The specimens were formalin-fixed and paraffin-embedded. The preoperative cervical secretions were collected and used to detect human papillomavirus (HPV) infection using hybrid capture generation II technology. 


\section{Cell culture}

HPV type 18 positive human cervical cancer cell line HeLa cells from ATCC cells were seeded onto 96well microplate at a density of $5 \times 10^{4}$ cells per well containing RPMI-advanced 1640 serum-free media (Gibco BRL, USA) with red phenol and antibiotic-antimycotic solution $(10,000$ units penicillin, $10 \mathrm{mg}$ streptomycin, and $25 \mu \mathrm{g} / \mathrm{ml}$ amphotericin B), supplemented with 1\% fetal bovine serum (Invitrogen, Carlsbad, CA) and $200 \mathrm{mM}$ of GlutaMAX (Invitrogen). The cells were incubated in $5 \%$ of $\mathrm{CO}_{2}$ and humidity saturation at $37^{\circ} \mathrm{C}$. Cells were harvested at 70\% confluence with Verseno solution (Tris base $25 \mathrm{mM}$, NaCL $136.8 \mathrm{mM}, \mathrm{KCl} 5.36$ mM, EDTA $1 \mathrm{mM} \mathrm{pH7.7)} \mathrm{and} \mathrm{washed} 3$ times in phosphate buffer saline.

\section{Drug treatment of Hela cells}

Cells were plated at $1 \times 10^{6}$ in 6-well dishes (Grier) and were allowed to attach for $24 \mathrm{~h}$. The cells were incubated with cisplatin at $15 \mathrm{mg} / \mathrm{l}$ or $25 \mu \mathrm{M}$ for $72 \mathrm{~h}$, a concentration which has been shown to be near the $\mathrm{IC}_{50}$ for apoptotic death of Hela cells [23, 24], or paclitaxel at $250 \mathrm{nM}$ [25], or carboplatin at $20 \mu \mathrm{M}$. The cells were then harvested with trypsin and washed twice with phosphate-buffered saline for subsequent uses for analysis of mRNAs and proteins. In separate experiments, Hela cells were pretreated with U0126 (10 $\mathrm{MM}$; 1,4-diamino-2,3-dicyano-1,4-bis[2-aminophenylthio] butadiene; Cell Signalling Technology, Inc) by incubation in the culture medium for $24 \mathrm{~h}$ before anti-cancer drug treatment. Or the cells were transfected with TAB1 siRNA or scrambled control siRNA sequence (Santa Cruz Biotech, Inc; Cat\#: sc-36600) by lipofectamine-2000 reagent (Invitrogen) for $24 \mathrm{~h}$ prior to anti-cancer drug treatment.

\section{Quantitative real-time RT-PCR analysis}

Quantitative real-time RT-PCR (qPCR) was used in conjunction with SYBR Green I incorporation methods to measure relative miRNA levels in our study. Total RNA samples were isolated from the cervical tumor tissue preparations and Hela cells with Trizol reagent. The reactions contained the primer sets specific for human miR-34a and miR-605, and a scrambled miRNA as a negative control. Taqman qPCR was employed for quantification of p53, Mdm2, and E2F1. qRT-PCR was performed on a thermocycler ABI Prism $® 7500$ fast (Applied Biosystems) for 40 cycles. Fold variations in expression of an mRNA between RNA samples were calculated. The threshold cycle $\left(C_{T}\right)$ is defined as the fractional cycle number at which the fluorescence passes the fixed threshold. Gapdh was used as an internal control for normalization. Fold or fractional changes of miRNA or mRNA were obtained by normalizing the values in cancer tissues to normal tissues.

\section{Western blot analysis}

The protein samples (cytosolic) were extracted for immunoblotting analysis. The protein content was measured by BCA Protein Assay Kit with bovine serum albumin as the standard. Protein sample $(\sim 80 \mu \mathrm{g})$ was fractionated by SDS-PAGE (12\% polyacrylamide gels) and transferred to PVDF membrane (Millipore, Bedford, MA). The sample was incubated overnight at $4^{\circ} \mathrm{C}$ with the primary antibodies in 1:1000: goat polyclonal anti-p53, anti-E2F1, anti-Mdm2, anti-phospho-ERK1/2, and anti-TAB1 (Cell Signaling Technology, Inc., Boston, MA, USA). Next day, the membrane was incubated with secondary antibodies (anti-rabbit IgG, HRP-linked antibody; Cell Signaling Technology) diluted in PBS for $3 \mathrm{~h}$ at room temperature. Finally, the membrane was rinsed with PBS before scanning using the Infrared Imaging System (LI-COR Biosciences). GAPDH was used as an internal control for equal input of protein samples, using anti-GAPDH antibody (Cell Signaling Technology, Inc.). Western blot bands were quantified using Imagequant 5.2 by measuring the band intensity for each group and normalizing to GAPDH. The final results are expressed as fold changes by normalizing the cancer data to the control values.

\section{Data analysis}

Data are expressed as mean \pm SD. Statistical analysis was carried out using SPSS Version 13.0 (SPSS Inc., Chicago, IL, USA). Survival curves were estimated by the Kaplan-Meier method, and compared using the log-rank test. Parameters that were significantly related to survival in univariate analysis were entered into the multivariate analysis. With a Cox proportional hazard model, multivariate analysis was done to identify the independent prognostic factors. For molecular analysis, paired comparisons between healthy and cancerous tissues were done by paired Student's $t$-test. A two-tailed $p<0.05$ was taken to indicate a statistically significant difference. 
Table 1. Patient characterization

\begin{tabular}{|c|c|c|}
\hline \multicolumn{3}{|l|}{ Clinical Characteristics } \\
\hline \multicolumn{3}{|l|}{ Age (years, range) } \\
\hline LRH Group & \multicolumn{2}{|c|}{$44.8 \pm 8.5(33-64)$} \\
\hline NAC+LRH Group & \multicolumn{2}{|c|}{$46.2 \pm 7.5(38-57)$} \\
\hline \multicolumn{3}{|l|}{ Clinical stage (FIGO) } \\
\hline LRH Group & IIb2 & 10 \\
\hline NAC+LRH Group & IIb2 & 11 \\
\hline \multicolumn{3}{|l|}{ Pathology } \\
\hline \multicolumn{3}{|l|}{ Squamous carcinoma } \\
\hline LRH Group & \multicolumn{2}{|l|}{7} \\
\hline NAC+LRH Group & \multicolumn{2}{|l|}{7} \\
\hline \multicolumn{3}{|l|}{ Adenocarcinoma } \\
\hline LRH Group & \multicolumn{2}{|l|}{2} \\
\hline NAC+LRH Group & \multicolumn{2}{|l|}{1} \\
\hline \multicolumn{3}{|c|}{ Adenosquamous carcinoma } \\
\hline LRH Group & \multicolumn{2}{|l|}{1} \\
\hline NAC+LRH Group & \multicolumn{2}{|l|}{3} \\
\hline
\end{tabular}

Table 2. Tumor size (diameter, $\mathrm{cm}$ ) with and without NAC, and before and after NAC

\begin{tabular}{llcc}
\hline & \multirow{2}{*}{ LRH } & \multicolumn{2}{c}{ NAC + LRH } \\
& Before NAC & After NAC \\
\hline 1 & 4.3 & 4.2 & 1.9 \\
2 & 6.6 & 6.9 & 4.1 \\
3 & 4.3 & 4.3 & 2.7 \\
4 & 4.7 & 5.2 & 2.0 \\
5 & 4.3 & 5.6 & 2.5 \\
6 & 4.5 & 5.8 & 2.2 \\
7 & 4.4 & 4.5 & 3.7 \\
8 & 5.2 & 4.3 & 3.3 \\
9 & 5.8 & 4.4 & 2.3 \\
10 & 4.3 & 4.2 & 2.4 \\
11 & \multicolumn{3}{l}{3.0} \\
\hline \multicolumn{2}{l}{ LRH: laparoscopical radical hysterectomy; } \\
NAC: neoadjuvant chemotherapy \\
\hline
\end{tabular}

\section{Results}

Reduction of tumor size and response to NAC

All individuals had completed at least one course of chemotherapy and undergone the corresponding evaluation (Table 1). The average diameter of the bulky tumors was $4.8 \pm 0.6$ $\mathrm{cm}(4.3-6.6 \mathrm{~cm})$ for the LRH group $(\mathrm{n}=10)$ and $4.9 \pm 0.7 \mathrm{~cm}(4.2-6.9 \mathrm{~cm})$ for the NAC+LRH group $(\mathrm{n}=11)$.

All 21 patients underwent MRI examination and measurement of the cervical lesion size prior to NAC and following the chemotherapy (Table 2). The diameter of tumors following chemotherapy in the NAC+LRH group was markedly reduced to $2.4 \pm 0.7 \mathrm{~cm}$ (Fig. $1)$, compared to that prior to chemotherapy $(P<0.01)$ and to that in the LRH group $(P<0.01)$.

As confirmed by pathological diagnosis following chemotherapy, there were 4 cases (36.4\%) of complete remission (CR), 6 cases (54.5\%) of partial remission (PR), only 1 SD 
Fig. 2. Western blot analysis of protein levels of p53 (A), Mdm2 (B) and E2F1 (C) with cervical cancer tissues for comparisons between LRH and NAC+LRH. LRH: laparoscopical radical hysterectomy and NAC: neoadjuvant chemotherapy. $\quad * * p<0.01 \quad \& \quad * * * p<0.001$ NAC+LRH vs LRH; Unpaired Student $t$-test; $\mathrm{n}=10$ for $\mathrm{LRH}$ and $\mathrm{n}=11$ for NAC+LRH.

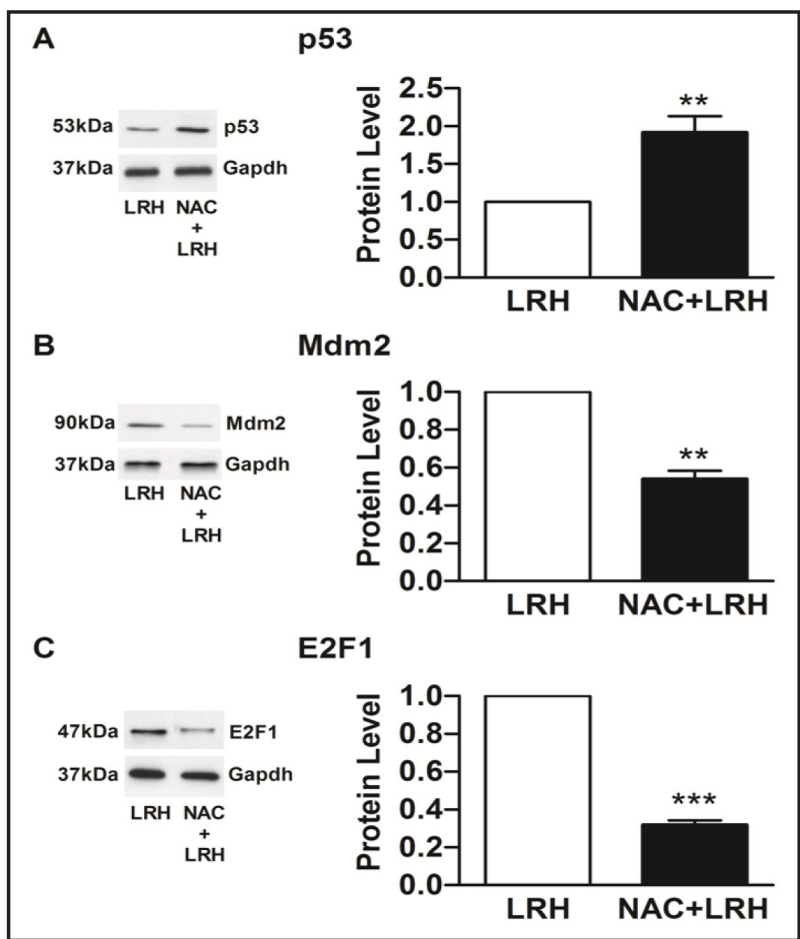

case $(9.1 \%)$ of stable disease (SD), and 0 case $(0 \%)$ of progression disease (PD). Thus, the chemotherapy response rate (i.e., CR + PR) was $90.9 \%$ (10/11) for the NAC+LRH group.

All 21 patients in the two groups completed the surgery with the surgical resection rate at $90.2 \%$. Of these 21 patients, only one case from the LRH group had lymph node metastasis after surgery, and no parametrial involvement and positive vaginal surgical margin cases were observed.

The median follow-up was 38 months. No cases of recurrence or metastasis after surgery have been observed in the NAC+LRH group as yet. In the LRH group, however, 2 recurrences and 1 metastasis were identified.

The 2-year disease-free survival rate was $80 \%$ for the LRH group and $90.9 \%$ for the NAC+LRH group ( $\mathrm{p}=0.066$; Fig. 1B). The 2 -year overall survival rate were $90 \%$ and $100 \%$ or the LRH group and the NAC+LRH group, respectively ( $p=0.057$; Fig. 1C).

\section{Expression of p53:miR-34a:E2F1 and p53:miR-605:Mdm2 signaling pathways}

Western blot analysis revealed remarkably higher levels of p53 tumor suppressor (Fig. 2A) and lower levels of E2F1 and Mdm2 oncoproteins in the NAC+LRH group compared to the LRH group without chemotherapy (Fig. 2B \& 2C). Coincidently and expectedly in light of the possible presence of the p53:miR-34a:E2F1 and the p53:miR-605:Mdm2 signaling pathways, the levels of miR-34a and miR-605 were considerably higher with NAC relative to without NAC (Fig. 3), as revealed by real-time RT-PCR quantification. The mRNA level of p53 was significantly elevated $(P<0.05)$, whereas that of E2F1 was downregulated $(P<0.05)$, following chemotherapy. Mdm2 mRNA expression remained unaltered by NAC $(P>0.05)$.

Cisplatin accounts for the enhanced p53 in NAC

We then continued to understand the mechanisms for the enhancement of p53:miR34a:E2F1 and p53:miR-605:Mdm2 signaling by NAC. In our study, the NAC consisted of three anti-cancer drugs cisplatin, paclitaxel, and carboplatin. Intriguingly, cisplatin is known to bind to and cause cross-linking of DNA to ultimately trigger apoptosis. Both cisplatin and its second generation drug carboplatin have been shown to increase p53 protein level reaching a threshold which leads to the initiation of apoptosis in Hela cells [26-28]. In contrast, TP53 


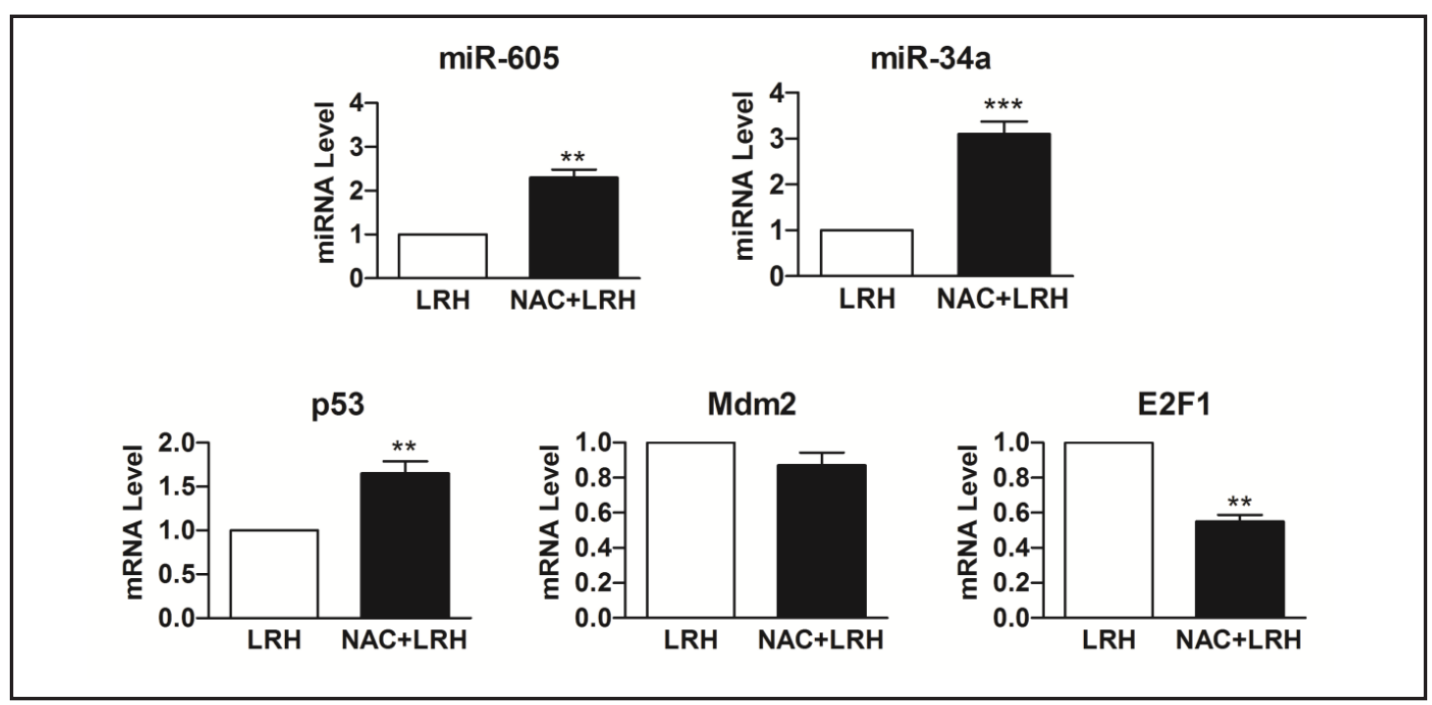

Fig. 3. Real-time RT-PCR quantification of miR-605, miR-34a, p53, Mdm2, and E2F1, showing the upregulation of miR-605, miR-34a and p53 mRNAs, and downregulation of E2F1, in patients with NAC compared to those without NAC. ${ }^{* *} p<0.01 \&{ }^{* * *} p<0.001$ NAC+LRH $v s$ LRH; Unpaired Student $t$-test; $\mathrm{n}=10$ for $\mathrm{LRH}$ and $\mathrm{n}=11$ for NAC+LRH.

Fig. 4. Comparison of effects of cisplatin, paclitaxel, and carboplatin on protein levels of p53 $(A), \mathrm{E} 2 \mathrm{~F} 1(B)$ and $\operatorname{Mdm} 2(C)$, by immunoblotting analysis in Hela human cervical cancer cells. Note that cisplatin produced greater increase in p53, and greater decreases in E2F1 and Mdm2

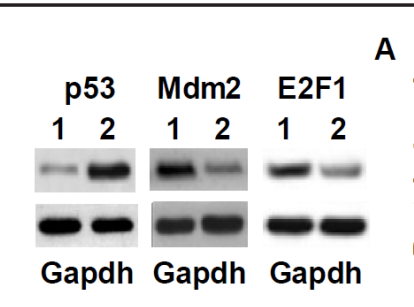

A

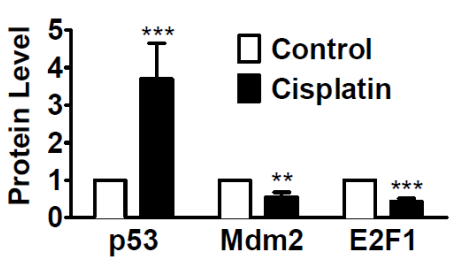
protein levels. 1: Control; 2: cisplatin $(A)$, paclitaxel $(B)$ or carboplatin (C). ${ }^{* *} p<0.01 \&{ }^{* * *} p<0.001$ Drug vs Control; ${ }^{\xi \xi \xi} p<0.001$ paclitaxel or carboplatin vs cisplatin; Unpaired Student t-test; $\mathrm{n}=5$ for each group.

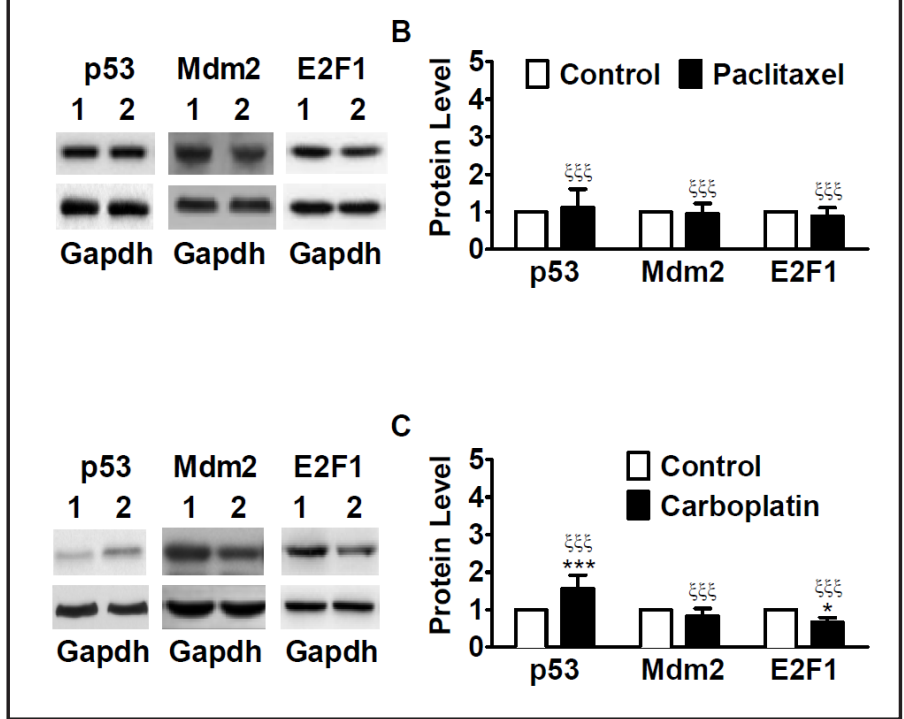

gene (encoding p53) status does not correlate with paclitaxel-induced cytotoxicity in various cancer cell lines with differing apoptotic pathways [29]. We therefore began our studies from comparing the effects of these three drugs individually on p53 protein levels. As depicted in Fig. 4A, cisplatin produced robust increase in p53, which was approximately 4-times greater 

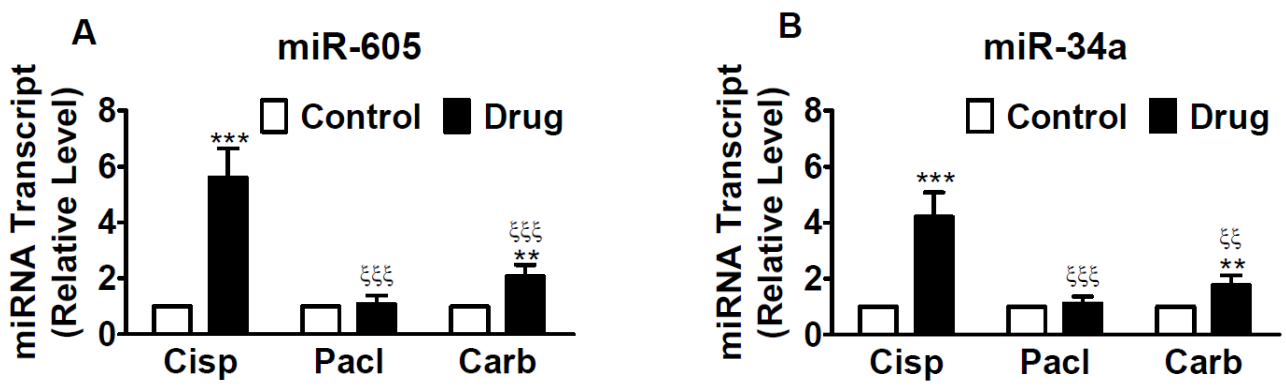

Fig. 5. Comparison of effects of cisplatin, paclitaxel, and carboplatin on miR-34a $(A)$ and miR-605 $(B)$ levels, by real-time RT-PCR quantification, in Hela human cervical cancer cells. Cisp: cisplatin; Pacl: paclitaxel; Carb: carboplatin. ${ }^{* *} p<0.01 \&{ }^{* * *} p<0.001$ Drug vs Control; ${ }^{\xi \xi} p<0.01 \&{ }^{\xi \xi \xi} p<0.001$ paclitaxel or carboplatin vs cisplatin; Unpaired Student $t$-test; $\mathrm{n}=5$ for each group.

Fig. 6. Effects of ERK1/2 inhibitor U0126 (A) or TAB1 siRNA on cisplatininduced upregulation of p53 protein $(B)$ and miR-605 $(C)$ and miR34a levels (D). Cells were pretreated with U0126 $(10 \mu \mathrm{M})$ or TAB1 siRNA before anti-cancer drug treatment. Cntrl: control; Cisp: cisplatin; siRNA: TAB1 SiRNA; Scrm: scrambled siRNA. ${ }^{* * *} p<0.001$ Cisplatin vs Control; ${ }^{\xi \xi \xi} p<0.001$ U0126 or TAB1 siRNA vs cisplatin; ${ }^{8 \delta \delta} p<0.001 \quad$ Scrambled siRNA vs TAB1 siRNA; Unpaired Student $t$-test; $\mathrm{n}=5$ for each group.

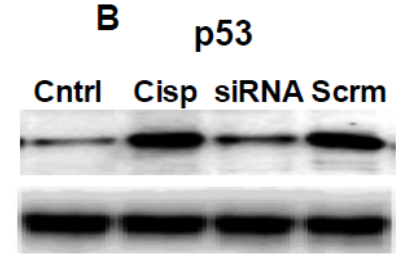

\section{Gapdh}

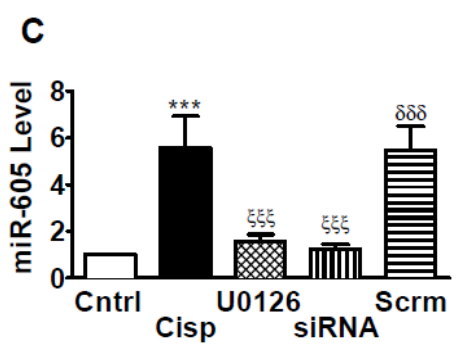

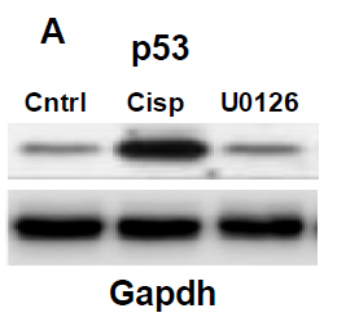
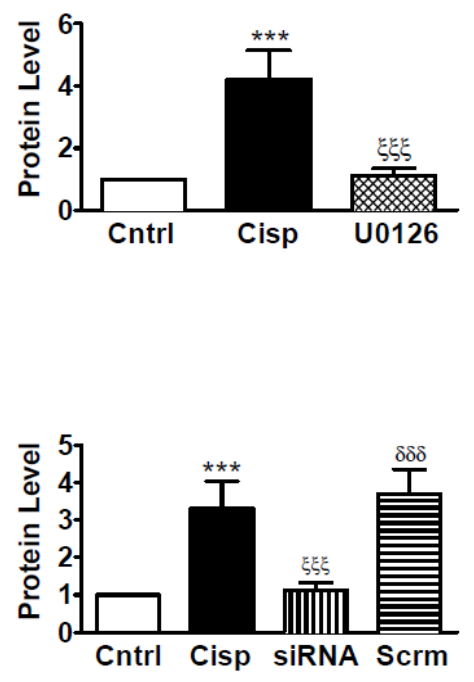

D

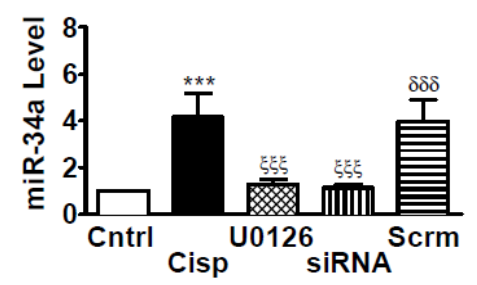

than carboplatin and 20-times greater than paclitaxel. Consistently, cisplatin reduced E2F1 and Mdm2 protein levels to much greater extents than the other two drugs (Fig. 4B \& 4C). Furthermore, cisplatin also remarkably upregulated miR-34a and miR-605, and so did carboplatin though to less degrees (Fig. 5). By comparison, paclitaxel did not affect the expression of these two miRNAs in Hela cells. These data indicate that cisplatin plays a critical role in promoting p53:miR-34a:E2F1 and p53:miR-605:Mdm2 signaling, paclitaxel may act mainly through other anti-cancer signaling mechanisms. 
Fig. 7. Changes of ERK1/2 activation and TAB1 expression. $(A)$ \& $(B)$ Verification of the efficacy of U0126 to inhibit ERK1/2 activity and TAB1 siRNA to knockdown TAB1, respectively, in human cervical cancer cell line HeLa cells. ${ }^{* * *} p<0.001$ vs Control, $\mathrm{n}=5$; $(C) \&(D)$ Verification of increased ERK1/2 activities (indicated by increased phopho-ERK1/2 protein) and TAB1 protein level, respectively, in cervical tumor tissues from $\mathrm{NAC}+\mathrm{LRH}$ patients $(\mathrm{n}=11)$ relative to LRH alone patients $(\mathrm{n}=10) .{ }^{* * *} p<0.001$ $\mathrm{NAC+LRH} v s$ LRH alone.

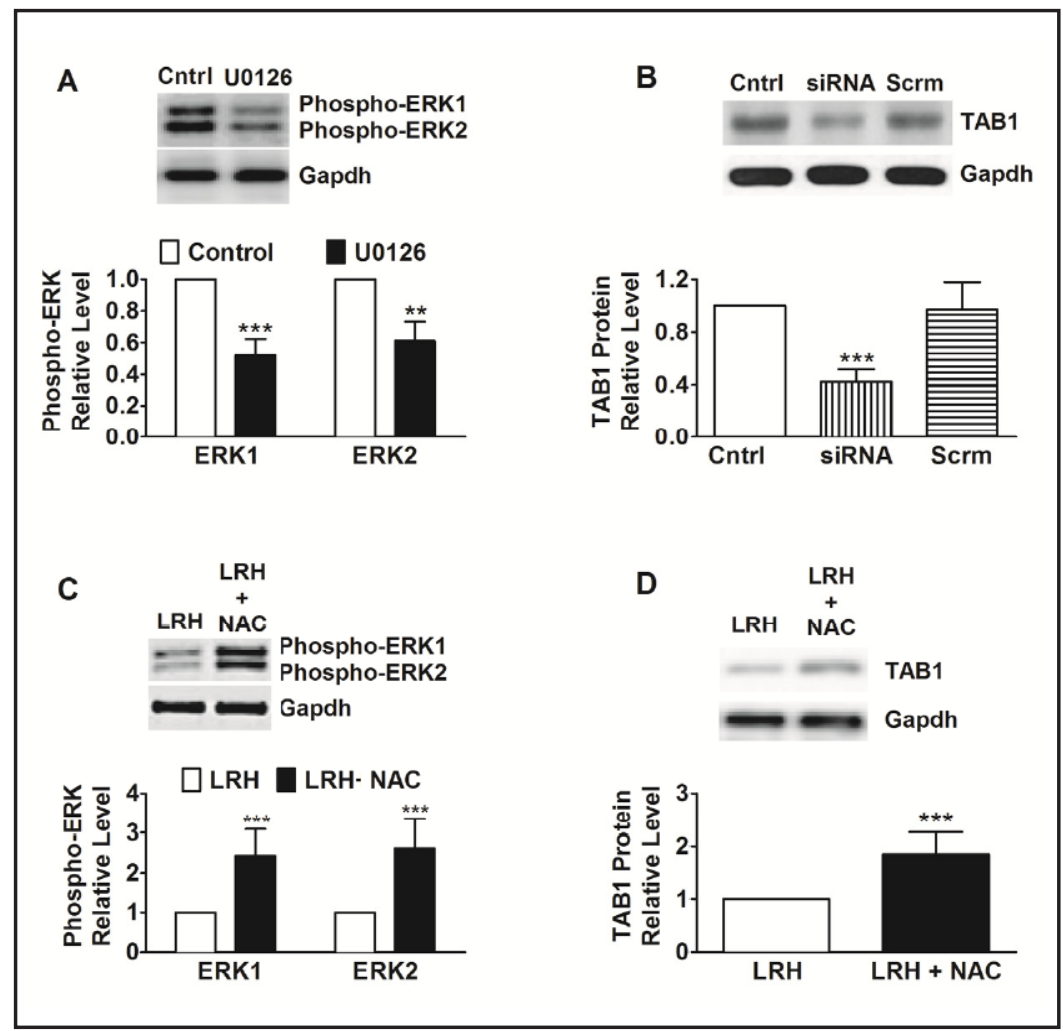

Mechanisms underlying the $p 53$ enhancing effects of cisplatin

We then went one step further towards understanding mechanisms for the enhancement of p53:miR-34a:E2F1 and p53:miR-605:Mdm2 signaling by NAC by exploiting how cisplatin favors p53 expression. It has been documented that cisplatin-induced ERK activation is an up-stream factor that enhances the p53 response to DNA damage caused by cisplatin [30, $31]$. On the other hand, cisplatin has also been demonstrated to cause cell death via TAB1 (transforming growth factor $\beta$-activated kinase 1 (TAK1)-binding protein 1) regulation of p53/MDM2/MDMX circuitry and cell death caused by cisplatin is mitigated by knockdown of TAB1 [24]. Hence, we assessed the effects of ERK1/2 inhibitor U0126 (10 $\mu \mathrm{M})$ or TAB1 siRNA on p53 protein levels. As expected, pretreatment with either of U0126 (incubation) or siRNA (transfection) substantially weakened the ability of cisplatin to upregulate p53 (Fig. 6A \& 6B). Similar effects on miR-34a and miR-605 were observed (Fig. 6C).

The efficacy of U0126 to inhibit ERK1/2 activity and of TAB1 siRNA to knockdown TAB1 was verified in human cervical cancer cell line HeLa cells (Fig. 7A \& 7B).

Finally, we also confirmed the increased ERK1/2 activities (indicated by increased phospho-ERK1/2 protein) and TAB1 protein level in cervical tumor tissues from NAC+LRH patients relative to LRH alone patients (Fig. 7C \& 7D).

\section{Discussion}

Here we performed direct parallel comparison of therapeutic outcomes and 2-year survival rates after laparoscopical radical hysterectomy (LRH) between the cervical cancer patients with and without neoadjuvant chemotherapy (NAC). Our data demonstrated that NAC+LRH produced apparently better therapeutic results than LRH alone: (1) the diameter of tumors following chemotherapy was substantially smaller in the NAC+LRH patients than in LRH patients; (2) None of the NAC+LRH patients had recurrence or metastasis after surgery, whereas out of $10 \mathrm{LRH}$ patients, 2 had recurrences and 1 had metastasis; (3) the 2-year disease-free and overall survival rates were apparently higher in the NAC+LRH 
group than in the LRH group. Furthermore, molecular biology analyses revealed that (1) the mRNA and protein levels of p53 were markedly increased in patients who received NAC than those who did not in cervical cancer tissues; the opposite was true for the levels of E2F1 and Mdm2; (2) the levels of miR-34a and miR-605 were considerably higher with NAC relative to without NAC; (3) among the three anti-cancer agent consisting of NAC in our study, cisplatin appears to play a more important role in upregulating p53 expression in Hela cervical cancer cells; and (4) inhibition of ERK1/2 or knockdown of TAB1 prevented the p53 upregulation by cisplatin. These findings suggest NAC as a rational approach for better treatment of cervical cancer in patients, partly due to enhancement of the p53:miR-34a:E2F1 positive feed-forward loop and the p53:miR-605:Mdm2 positive feedback loop. Moreover, activation of ERK1/2 and TAB1 by cisplatin likely accounts for its ability to upregulate p53 so as to stimulate p53:miR-34a:E2F1 positive feed-forward loop and the p53:miR-605:Mdm2 signaling, contributing to improved therapeutic outcomes with NAC.

Concurrent chemoradiotherapy and LRH have recently been regarded as the standard of treatment for high risk tumors [32-35]. Evidence exists for the use of cisplatin based combination chemotherapy to improve overall survival in women with advanced cervical cancers [36-42]. Experiences with neoadjuvant chemotherapy for poor risk cervical carcinomas suggested that cisplatin-based combination chemotherapy was effective not only in local tumor and may also reduce the incidence of lymph node metastasis and even eradicate microscopic metastasis in distant organs [32-34]. However, in contrast to these results, Katsumata et al. reported a randomized, controlled study of stage Ib2-IIb cervical cancer, which showed no significant difference between a NAC-combined surgery group and the surgery alone group in terms of resection and survival rate [43]. This discrepancy is attributed, in the authors' opinion, to the differences of chemotherapy regimens, courses and case selections. Early clinical investigations on NAC have primarily been focused on cisplatin-based chemotherapy, and numerous studies have confirmed that the response rate of cisplatin-combined duplex treatment is superior to cisplatin monotherapy [44]. The advantages of NAC may involve its ability to reduce tumor size, improve resection rate, and limit lymph node metastasis rate. In the present study, the NAC includes three agents: cisplatin, paclitaxel, and carboplatin, and this regimen indeed manifested these advantages of combination chemotherapy.

The primary cause of cervical cancer persistent infection by the human papillomavirus (HPV), which induces degradation of tumor suppressor p53 [6-10]. Consistent with this mechanism, the majority of cervical cancer cells are found to possess a wild-type p53 gene, but the protein levels are extremely low. Correction of this aberrant insufficiency of p53 is a rational strategy for the cure of cervical cancer. To achieve this goal, one approach would be to destroy the HPV E6 oncoprotein so as to release p53 from degradation. Alternatively, p53 can be activated in terms of its expression and function by other approaches. For instance, an interference with the negative feedback inhibition of p53 by oncoprotein Mdm2 may be able to upregulate and activate p53; one way to achieve this is to upregulate miR-605 to repress Mdm2. Our data provide a piece of evidence for the validity of this approach. We found here that miR-605 was robustly upregulated following NAC. On the basis of the p53:miR34a:E2F1 positive feed-forward loop and the p53:miR-605:Mdm2 positive feedback circuit, it is not unreasonable to speculate that upregulation of miR-605 produces repressive effects on Mdm2, resulting in upregulation of p53, which in turn transactivates miR-34a to downregulate E2F1, leading to tumor inhibition. Our findings of upregulation of miR-605, miR-34a, and p53, and of concurrent downregulation of Mdm2 and E2F1 fit well to this signaling pathway. The present study therefore helps us better understand the mechanisms for NAC of cervical cancer.

P53 can be stimulated for its expression and accumulation by any stress that causes DNA damaging. Cisplatin is known for its ability to bind to and cause cross-linking thereby damage of DNA. It is no wonder that it is able to cause p53 expression and accumulation, as shown in the present study and in many previous studies as well [26-28]. Carboplatin is a second generation of cisplatin differing from cisplatin in that it has a bidentate dicarboxylate 
(CBDCA) ligand in place of the two chloride ligand. Carboplatin has gained popularity in clinical treatment due to its vastly reduced side-effects compared to its parent compound cisplatin. However, it exhibits lower reactivity and slower DNA binding kinetics, although it forms the same reaction products in vitro at equivalent doses with cisplatin $[45,46]$. Unlike cisplatin, carboplatin may be susceptible to alternative mechanisms. Some results show that cisplatin and carboplatin cause different morphological changes in MCF-7 cell lines while exerting their cytotoxic behaviour [47]. These properties of carboplatin may explain for its smaller effects on p53 expression as revealed in this study. Paclitaxel is a mitotic inhibitor and it stabilizes microtubules and as a result, interferes with the normal breakdown of microtubules during cell division $[48,49]$. This mechanism of paclitaxel action is consistent with its lack of direct effects on p53 in the published studies [29] and also in the present study.

It should be noted that our study only included a total of 21 patients and the sample size is too small to allow us to have accurate comparison between the treatments with and without NAC and to make conclusive note on the benefit of NAC for LRH treatment of cervical cancer. Moreover, in terms of the mechanistic insight of the NAC, our study is also preliminary, merely providing expression data without using gain- and loss-of-function approaches to confirm our notion about the involvement of the p53:miR-34a:E2F1 and p53:miR-605:Mdm2 pathways. Nonetheless, our study lays the groundwork for future studies to test the thoughts. Also precaution must be taken when interpreting our results on the drug effects on p53 expression. Only one concentration of each agent was tested in Hela cells, and the relative concentrations of the three agents may not be in the same proportion of these drugs in NAC. The conclusion that cisplatin plays the main role in stimulating increasing p53:miR34a:E2F1 and p53:miR-605:Mdm2 signaling may not hold true, as increasing concentrations of paclitaxel and carboplatin may produce similar effects as cisplatin. Nevertheless, our data are consistent with previous studies by other groups showing the effect of cisplatin and the lack of effect of paclitaxel on p53. Perhaps, the key point on this regard is that our study revealed the benefit afforded by NAC is related to p53 stimulation via ERK1/1 and TAB1.

Collectively, this study combined clinical observations and experimental investigations to study the mechanisms for the beneficial effects of NAC in the surgical treatment of patients with cervical cancer. The findings from this study indicate that NAC improves LRH treatment of cervical cancer and suggest that the p53:miR-34a:E2F1 positive feed-forward circuit and the p53:miR-605:Mdm2 positive feedback loop are operating to confer the beneficial effects of NAC. Among the three anti-cancer drugs, cisplatin appears to play the major role in promoting the p53:miR-34a:E2F1 and p53:miR-605:Mdm2 signaling pathways. This action of cisplatin is likely conferred by its ability to interact with ERK1/2 and TAB1.

\section{Conflict of Interests}

The authors declare no competing financial interests.

\section{Acknowledgements}

This study was supported in part by a grant from the National Natural Science Foundation of China for Young Scholars (Grant No: 81202047).

\section{References}

1 Arbyn M, Castellsagué X, de Sanjosé S, Bruni L, Saraiya M, Bray F, Ferlay J: Worldwide burden of cervical cancer in 2008. Ann Oncol 2011;22:2675-2686.

-2 Creasman WT, Odicino F, Maisonneuve P, Beller U, Benedet JL, Heintz AP, Ngan HY, Sideri M, Pecorelli S: Carcinoma of the corpus uteri. J Epidemiol Biostat 2001;61:47-86.

3 Parkin DM, Bray F, Ferlay J, Prisani P: Global cancer statistics, 2002. CA Cancer J Clin 2005;55:74-108. 
4 Fu Y, Reagan J: Pathology of the Uterine Cervix, Vagina and Vulva. Major Problems in Pathology. Philadelphia, W.B. Saunders and Co, 1989, pp288-335.

5 Pisani P, Bray F, Parkin DM: Estimates of the world-wide prevalence of cancer for 25 sites in the adult population. Int J Cancer 2002;97:72-81.

6 6 Tommasino M, Accardi R, Caldeira S, Dong W, Malanchi I, Smet A, Zehbe I: The role of TP53 in Cervical carcinogenesis. Hum Mutat 2003;21:307-312.

-7 Habbous S, Pang V, Eng L, Xu W, Kurtz G, Liu FF, Mackay H, Amir E, Liu G: p53 Arg72Pro polymorphism, HPV status and initiation, progression, and development of cervical cancer: a systematic review and metaanalysis. Clin Cancer Res 2012;18:6407-6415.

8 Arends MJ, Buckley CH, Wells M: Aetiology, pathogenesis, and pathology of cervical neoplasia. J Clin Pathol 1998;51:96-103.

9 Yaziji H, Gown AM: Immunohistochemical analysis of gynecologic tumors. Int J Gynecol Pathol 2001;20:6478.

10 Tan S, de Vries EG, van der Zee AG, de Jong S: Anticancer drugs aimed at E6 and E7 activity in HPV-positive cervical cancer. Curr Cancer Drug Targets 2012;12:170-184.

-11 Adewole IF, Benedet JL, Crain BT, Follen M: Evolving a strategic approach to cervical cancer control in Africa. Gynecol Oncol 2005;99:S209-212.

12 Emembolu JO, Ekwempu CC: Carcinoma of the cervix uteri in Zaria: etiological factors. Int J Gynaecol Obstet 1988;26:265-269.

13 Hacker NF, Friedlander ML: Cervical cancer; in Berek JS, Hacker NF (eds): Berek \& Hacker's gynecologic oncology. 5th ed. Philadelphia, Lippincott Williams \& Wilkins, 2010, pp341-395.

14 Bidus MA, Elcas JC: Cervical and vaginal cancer; in Berek JS, Novak E (eds): Berek \& Novak's gynecology. 14th ed. Philadelphia, Lippincott Williams \& Wilkins, 2007, pp1403-1456.

15 Boyce J, Fruchter RG, Nicastri AD, Ambiavagar PC, Reinis MS, Nelson JH Jr: Prognostic factors in stage I carcinoma of the cervix. Gynecol Oncol 1981;12:154-165.

16 Delgado G, Bundy B, Zaino R, Sevin BU, Creasman WT, Major F: Prospective surgical-pathological study of disease-free interval in patients with stage IB squamous cell carcinoma of the cervix: a Gynecologic Oncology Group study. Gynecol Oncol 1990;38:352-357.

$\checkmark 17$ Raver-Shapira N, Marciano E, Meiri E, Spector Y, Rosenfeld N, Moskovits N, Bentwich Z, Oren M: Transcriptional activation of miR-34a contributes to p53-mediated apoptosis. Mol Cell 2007;26:731-743.

$\rightarrow 18$ Rokhlin OW, Scheinker VS, Taghiyev AF, Bumcrot D, Glover RA, Cohen MB: MicroRNA-34 mediates ARdependent p53-induced apoptosis in prostate cancer. Cancer Biol Ther 2008;7:1288-1296.

19 Chang TC, Wentzel EA, Kent OA, Ramachandran K, Mullendore M, Lee KH, Feldmann G, Yamakuchi M, Ferlito M, Lowenstein CJ, Arking DE, Beer MA, Maitra A, Mendell JT: Transactivation of miR-34a by p53 broadly influences gene expression and promotes apoptosis. Mol Cell 2007;26:745-752.

20 Barak Y, Juven T, Haffner R, Oren M: mdm2 expression is induced by wild type p53 activity. EMBO J 1993;12:461-468.

21 Harris SL, Levine AJ: The p53 pathway: positive and negative feedback loops. Oncogene 2005;24:28992908.

-22 Xiao J, Lin H, Luo X, Luo X, Wang Z: miR-605 joins p53 network to form a p53:miR-605:Mdm2 positive feedback loop in response to stress. EMBO J 2011;30:524-532.

-23 Su XY, Yin HT, Li SY, Huang XE, Tan HY, Dai HY, Shi FF: Intervention effects of nedaplatin and cisplatin on proliferation and apoptosis of human tumour cells in vitro. Asian Pac J Cancer Prev 2012;13:4531-4536.

24 Zhu Y, Regunath K, Jacq X, Prives C: Cisplatin causes cell death via TAB1 regulation of p53/MDM2/MDMX circuitry. Genes Dev 2013;27:1739-1751.

-25 Hennequin C, Giocanti N, Favaudon V: Interaction of ionizing radiation with paclitaxel (Taxol) and docetaxel (Taxotere) in HeLa and SQ20B cells. Cancer Res 1996;56:1842-1850.

26 Siemer S, Ornskov D, Guerra B, Boldyreff B, Issinger OG: Determination of mRNA, and protein levels of p53, MDM2 and protein kinase CK2 subunits in F9 cells after treatment with the apoptosis-inducing drugs cisplatin and carboplatin. Int J Biochem Cell Biol 1999;31:661-670.

-27 Huang H, Huang SY, Chen TT, Chen JC, Chiou CL, Huang TM: Cisplatin restores p53 function and enhances the radiosensitivity in HPV16 E6 containing SiHa cells. J Cell Biochem 2004;91:756-765.

28 Okamoto S, Jiang Y, Kawamura K, Shingyoji M, Fukamachi T, Tada Y, Takiguchi Y, Tatsumi K, Shimada H, Hiroshima K, Kobayashi H, Tagawa M: Zoledronic acid produces combinatory anti-tumor effects with cisplatin on mesothelioma by increasing p53expression levels. PLoS One 2013;8:e60297. 
29 Takahashi M, Kigawa J, Minagawa Y, Itamochi H, Shimada M, Kamazawa S, Sato S, Akeshima R, Terakawa $\mathrm{N}$ : Sensitivity to paclitaxel is not related to p53-dependent apoptosis in ovarian cancer cells. Eur J Cancer 2000;36:1863-1868.

-30 DeHaan RD, Yazlovitskaya EM, Persons DL: Regulation of p53 target gene expression by cisplatin-induced extracellular signal-regulated kinase. Cancer Chemother Pharmacol 2001;48:383-388.

- 31 Persons DL, Yazlovitskaya EM, Pelling JC: Effect of extracellular signal-regulated kinase on p53 accumulation in response to cisplatin. J Biol Chem 2000;275:35778-35785.

-32 Benedet JL, Bender H, Jones H, Ngan HY, Pecorelli S: FIGO staging classifications and clinical practice guidelines in the management of gynecologic cancers. FIGO Committee on Gynecologic Oncology. Int J Gynaecol Obstet 2000;70:209-262.

-33 Iwasaka T, Kamura T, Yokoyama M, Matsuo N, Nakano H, Sugimori H: Adjuvant chemotherapy after radical hysterectomy for cervical carcinoma: a comparison with effects of adjuvant radiotherapy. Obstet Gynecol 1998;91:977-981.

-34 Takeshima N, Umayahara K, Fujiwara K, Hirai Y, Takizawa K, Hasumi K: Treatment results of adjuvant chemotherapy after radical hysterectomy for intermediate- and high-risk stage IB-IIA cervical cancer. Gynecol Oncol 2006;103:618-622.

-35 Tattersall MH, Ramirez C, Coppleson M: A randomized trial of adjuvant chemotherapy after radical hysterectomy in stage Ib-IIa cervical cancer patients with pelvic lymph node metastases. Gynecol Oncol 1992;46:176-181.

- 36 Alberts DS, Garcia D, Mason-Liddil N: Cisplatin in advanced cancer of the cervix: an update. Semin Oncol 1991;18:11-24.

-37 Runowicz CD, Wadler S, Rodriguez-Rodriguez L, Litwin P, Shaves M, O'Hanlan KA, Goldberg GL, Tomaino CT, Byrnes R: Concomitant cisplatin and radiotherapy in locally advanced cervical carcinoma. Gynecol Oncol 1989;34:395-401.

- 38 Al-Saleh E, Hoskins PJ, Pike JA, Swenerton KD: Cisplatin/ etoposide chemotherapy for recurrent or primarily advanced cervical carcinoma. Gynecol Oncol 1997;64:468-472.

39 Olive ST, Kiser WR: Diagnosis of appendicitis. J Am Board Fam Pract 1996;9:306-307.

40 Bonomi P, Blessing J, Ball H, Hanjani P, DiSaia PJ: A phase II evaluation of cisplatin and 5-fluorouracil in patients with advanced squamous cell carcinoma of the cervix: a Gynecologic Oncology Group Study. Gynecol Oncol 1989;34:357-359.

41 Namkoong SE, Park JS, Kim JW, Bae SN, Han GT, Lee JM, Jung JK, Kim SJ: Comparative study of the patients with locally advanced stages I and II cervical cancer treated by radical surgery with and without preoperative adjuvant chemotherapy. Gynecol Oncol 1995;59:136-142.

-42 Alberts DS, Kronmal R, Baker LH, Stock-Novack DL, Surwit EA, Boutselis JG, Hannigan EV: Phase II randomized trial of cisplatin chemotherapy regimens in the treatment of recurrent or metastatic squamous cell cancer of the cervix: a Southwest Oncology Group Study. J Clin Oncol 1987;5:1791-1795.

43 Katsumata N, Yoshikawa H, Hirakawa T, Saito T, Kuzuya K, Fujii T, Hiura M, Tsunematsu R, Fukuda $\mathrm{H}$, Kamura T: Phase III randomized trial of neoadjuvant chemotherapy (NAC) followed by radical hysterectomy (RH) versus RH for bulky stage I/II cervical cancer. ASCO Annu Meet Proc 2006;24: abs. 5013.

44 Monk BJ, Huang HQ Cella D, Long HJ $3^{\text {rd }}$ : Gynecologic Oncology Group Study: Quality of life outcomes from a randomized phase III trial of cisplatin with or without topotecan in advanced carcinoma of the cervix: a Gynecologic Oncology Group Study. J Clin Oncol 2005;23:4617-4625.

45 Wheate NJ, Walker S, Craig GE, Oun R: The status of platinum anticancer drugs in the clinic and in clinical trials. Dalton Trans 2010;39:8113-8127.

46 Fujita Y, Seki N, Inoue K, Miyazawa T, Eguchi K: A comparison of cisplatin and carboplatin: a short review. West Indian Med J 2010;59:115.

47 Natarajan G, Malathi R, Holler E: Increased DNA-binding activity of cis-1,1-cyclobutanedicarboxylatodi ammineplatinum(II) (carboplatin) in the presence of nucleophiles and human breast cancer MCF-7 cell cytoplasmic extracts: activation theory revisited. Biochem Pharmacol 1999;58:1625-1629.

48 Jordan MA, Wilson L: Microtubules as a target for anticancer drugs. Nat Rev Cancer 2004;4:253-265.

49 Fuchs, David A and Johnson, Randall K: Cytologic evidence that taxol, an antineoplastic agent from Taxus brevifolia, acts as a mitotic spindle poison. Cancer Treat Rep 1978;62:1219-1222. 